\title{
Green Synthesis of Silver Nanoparticles using Medicinal Plants Berberis asiatica and Cassia fistula and Evaluation of Antioxidant and Anti-bacterial Activities
}

\author{
Deegendra Khadka, ${ }^{+}$Rachana Regmi, ${ }^{1}$ Mitesh Shrestha ${ }^{2}$ and Megha Raj Banjara ${ }^{3}$ \\ ${ }^{1}$ Nepal Academy of Science and Technology, Khumaltar, Lalitpur, Nepal \\ ${ }^{2}$ Research Institute for Bioscience and Biotechnology, Ekantakuna, Lalitpur, Nepal \\ ${ }^{3}$ Central Department of Microbiology, Tribhuvan University, Kirtipur, Kathmandu, Nepal
}

\section{*CORRESPONDENCE:}

Deegendra Khadka

Nepal Academy of Science and

Technology, Khumaltar, Lalitpur, Nepal

Email: deegendrakhadka@gmail.com

ISSN : 2382-5359(Online), 1994-1412(Print)

DOI:

https://doi.org/10.3126/njst.v20i1.39384

\section{ACCESS THE ARTICLE ONLINE}

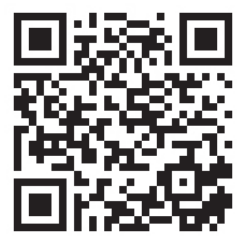

\section{CONFILICT OF INTEREST: None}

Copyright: The Author(s) 2020. This is an open access article under the CC BY license.

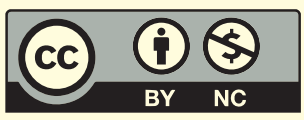

\begin{abstract}
The application of silver nanoparticles in various sectors including health related field is remarkably profound. Nowadays, the research of synthesizing metal nanoparticles (MNPs) using plant extracts is fascinating field as it offers the eco-friendly and cost-effective method for nanoparticle synthesis. In this study, we synthesized silver nanoparticles (AgNPs) using methanolic extract of B.asiatica and C. fistula regarding their ethnomedical importance.The synthesized AgNPs were characterized by UV-Visible spectroscopy, Fourier transform infrared spectroscopy (FTIR) and X-ray diffractometer (XRD). UV-vis spectroscopy exhibited the characteristic Surface Plasmon Peak of silver nanoparticle $420 \mathrm{~nm}$.FTIR data were measured to get a preliminary idea on the functional groups responsible for the stabilization of AgNPs. XRD data confirmed the natural crystal structure with a face centered cubic of AgNPs. The antibacterial activity of biosynthesized AgNPs was assessed by testing promptly available gram-positive Staphylococcus aureus and gram-negative Escherichia coli bacterial strain and antioxidant activity was calculated by DPPH assay. The overall outcomes of the studies concluded that the application of the biogenic synthesis of AgNPs of B. asiaticaas an antioxidant and antibacterial agent is more potent showing $\mathrm{IC}_{50}$ value $65.1 \pm 1.30 \mu \mathrm{g} / \mathrm{mL}$ and the highest zone of inhibition $15 \mathrm{~mm}$ in diameter against $S$. aureus.
\end{abstract}

Keywords: Berberis asiatica, cassia fistula, biosynthesis, silver nanocolloid, anti-bacterial potential

\section{INTRODUCTION}

Pharmaceutical industries have been using metal nanoparticles (MNPs) tremendously in the manufacturing of ointments and creams to get relief from burns and wounds associated with infections (Satyavani et al. 2011). Synthesizing MNPs using plant extracts is the most adequate biosynthetic method because of being eco-friendly and cost-efficient. Currently, several plant parts like leaf, fruit, stem, bark and seed extracts in different solvents are being productively applied for the synthesis of MNPs (Mittal et al. 2013). In the ratio of other MNPs, silver nano-particles (AgNPs) are more demanding due to their strong inhibitory activity against a number of microorganisms (Yugal Kishore Mohanta \& Behera 2014). Having significant anti-microbial activity, the phyto-mediated AgNPs are widely implemented in food packaging, food and seed preservation, bio-fertilizers, cosmetics, medicines and so on (Marambio-Jones \& Hoek 2010; Dipankar \& Murugan 2012). The implication of AgNPs in the control of microbial and treating cancer as potential drug carrier has presently earned substantial attention (Nayak et al. 2016). 
Several established protocols are available to synthesize AgNPs viz. chemical and photochemical reaction in reverse micelles, thermal decomposition of silver compound, radiation based, microwave-assisted process, sono-chemical process and currently becoming familiar "green chemistry technology" (Iravani et al. 2014). Green synthesis is more significant in the comparison of physical and chemical approaches because of being cost effective and environmentally compatible and problem free while scaling up for producing in large quantity without applying high pressure, energy, temperature and toxic chemicals (Kharissova et al. 2019). In the green synthesis of AgNPs, the two methods either using biological microorganisms or plant extracts based have been expended but phytomediated is more advantageous as it doesn't necessitate the tedious processes of preserving the cell cultures and aseptic conditions (Loo et al. 2018).

The articles describing different methods applied in the green synthesis of AgNPs by using plant extracts have been published in the renewed journals. However, there is still a demand for such procedures which are environmentally friendly, commercially survival and economically sustainable to synthesize plant based AgNPs (Beyene et al. 2017). Regarding current trends in the synthesis of AgNPs, using plants and plant based products are being a fascinating research domain (Khandel et al. 2018). A variety of ethno-medicinal plants are immensely used in the production of herbal medicine and their bioactive compounds are demanded in extremes for healthcare and nutritional products as a principal ingredient. In our study, B. asiatica and C. fistula were chosen concerning their medicinal values after literature survey. Berberis genius is massively used in many medical fieldssince ancient periods for the treatment of wound healing, eye disease fever, kidney and gall balder stones, jaundice, vomiting during pregnancy, rheumatism, and several other illnesses. Several bioactive compounds found on the roots and leaf of B. asiatica areBerberine, Colunbamamine, Jatrrohirine, Karachine, Oxyacanthine, Oxyberberine, Palmitine, Taxilamine and Tetrahydropalmitine (Bhardwaj \& Kaushik 2012). In Ayurveda, B. asiaticais used singly or in combination with other medicinal plants for healing a variety of ailments such as enlargement of spleen, leprosy, rheumatism, fever, morning or evening sickness and snakebite (Belwal et al. 2017). AgNPs synthesized from the steam bark extract of $B$. asiatica showed potent antioxidant and antimicrobial activities (Bhandari et al. 2000). Thus we use the leaf of $B$. asiatica for the synthesis of AgNPs. The AgNPs synthesized using India dwelling C. fistula has established effective results for antioxidant, antimicrobial and anticancer activities (Mohanta et al. 2016).No further literatures are available describing antioxidant and antibacterial activities and the green synthesis of AgNPS using Nepal inhabiting C. fistula medicinal plant. So, we synthesized AgNPs using $C$. fistula and reported its antioxidant and antibacterial activities for the first time.

\section{MATERIALS AND METHODS}

\subsection{Materials}

Plant specimens were collected from Kailali and Gandaki districts of Nepal in October 2018. Table 1 contains descriptive information of the plants used in the study. Chemicals and reagents consumed in the experiment are of molecular grade. Water used during experiment was PCRgrade obtained from Milli-Q water purification. Origin Pro 18 and Graph Pad Prisma 8 software were used for data analysis.

Table 1 Details of the medicinal plants used in the experiment

\begin{tabular}{cccccc}
\hline Scientific Name & Local name & Locality & Altitude & Parts used & \% yield (Methanol) \\
\hline Berbarisasiatica & Chuthro & $\begin{array}{c}\text { Low camp, Mardi } \\
\text { Himal }\end{array}$ & $3200 \mathrm{~m}$ & Leaves & 15.5 \\
\hline Cassia fistula & Rajbrikshya & Dhangadi, Kailali & $109 \mathrm{~m}$ & Leaves & 12.8 \\
\hline
\end{tabular}

\subsection{Extract Preparation}

The medicinal plants were collected (Table 1) and washed thoroughly with distilled water then dried at room temperature for a month. The dried plants material was pulverized by a grinder and extracted in methanol by soxhlet extraction process. Thus obtained extract was concentrated using a rota evaporator and made completely dried keeping at room temperature. The extract was stored at $-20{ }^{\circ} \mathrm{C}$ for further studies.

\subsection{Biosynthesis of Silver Nanoparticle}

A mixture was prepared by adding $100 \mathrm{mg}$ of plant extract to $100 \mathrm{~mL}$ of $1 \mathrm{mM}$ silver nitrate solution. The color of the mixture was changed from brown to yellow after incubating at the room temperature for a day. The reduction of $\mathrm{Ag}+$ was examined by scanning spectrophotometer and sample absorbance was taken in the range of 350$700 \mathrm{~nm}$ at minimum scanning rate. Distilled water (RO) taken from Milli-Q was used as the blank reference in the 
baseline correction of the spectrometer. After 24 hours, the prepared AgNPs was centrifuged at $9000 \mathrm{rpm}$ for $20 \mathrm{~min}$ at $25^{\circ} \mathrm{C}$ (Ahmed et al. 2016). The supernatant liquid was discarded and obtained pellet was washed with distilled water. The purified AgNPs were dried in a hot air oven at $25-26{ }^{\circ} \mathrm{C}$ and stored in an eppendorf tube at $4{ }^{\circ} \mathrm{C}$ until further requirement.

\subsection{Characterization of AgNPs}

\subsubsection{UV-Vis spectroscopy}

Green synthesized AgNPs solution in deionized water was monitored by using Agilent technologies, Cary-60 UV-Vis spectrophotometer. The absorption spectra of the nanoparticles were measured between 300 to $700 \mathrm{~nm}$ in a quartz cuvette taking water as reference for the base line correction (Elamawi et al. 2018).

\subsubsection{Fourier Transform-Infrared (FTIR) spectroscopy}

The FTIR spectrum of biosynthesized AgNPs was recorded in FTIR spectrometer to determine the presence of different functional groups found in the sample. Initially, the background screening of potassium bromide (KBr) was done. Then, a KBr pellet was made with AgNPs and scanned again. The spectra showed different frequencies and hence the frequencies were analyzed using infrared and Raman spectroscopy table. All measurements were carried out in the range of $400-4000 \mathrm{~cm}^{-1}$ at a resolution of $4 \mathrm{~cm}^{-1}$ by using IRTracer-100 FTIR spectrophotometer, Shimadzu to identify the present functional groups on the synthesized AgNPs.

\subsubsection{X-ray diffraction (XRD)}

The pellets of the phyto-mediated AgNPs was prepared in autoclaved Milli-Q water at 10,000 rpm for 15 minutes. Thus prepared pellets were oven dried at $50{ }^{\circ} \mathrm{C}$ to analyze the particle size by XRD. The nature of AgNPs was studied by using Bruker D2 Phaser Diffractometer, which was observed at $30 \mathrm{kV}$ voltage and $10 \mathrm{~mA}$ current with a monochromatic $\mathrm{Cu} K \alpha$ radiation $\operatorname{source}(\lambda=0.15418 \mathrm{~nm})$ having $2 \theta$ angles ranging from $20^{\circ}$ to $80^{\circ}$.

\subsection{Antioxidant Activity}

\subsubsection{Free radical scavenging ability on 2, 2-diphenyl-2-picrylhydrazyl (DPPH)}

Ascorbic acid in the concentration of 10, 20,60, 80, 100,120 and $150 \mu \mathrm{g} / \mathrm{mL}$ was prepared as reference and sample solutions of crude plant extract and AgNPs of the concentration 2000,1500,1000,500, 250, 125 and $62.5 \mu \mathrm{g} / \mathrm{mL}$ were prepared in DMSO. DPPH solution of
$0.01 \mathrm{mM}$ was made in absolute methanol.100 $\mu \mathrm{L}$ of $0.1 \mathrm{mM}$ DPPH solution was added to $100 \mu \mathrm{L}$ of each concentration of the sample and ascorbic acid solution. The mixtures were prepared in 96 well plates and were kept in dark for 30 minutes. Similarly, $200 \mu \mathrm{L}$ of $0.1 \mathrm{mM}$ DPPH solution was used as control. Thirty minutes later, the absorbance was taken at $517 \mathrm{~nm}$. The capability to scavenge the DPPH radical was calculated by using the following equation (Nagaich et al. 2016):

$$
\text { Percentage Scavenging }=\times 100
$$

$\mathrm{A}_{\mathrm{o}}=$ Absorbance of DPPH solution and $\mathrm{A}_{\mathrm{t}}=$ Absorbance of test or reference sample

The percentage scavenging was plotted against concentration and regression equation to obtain $\mathrm{IC}_{50}$ (concentration required to inhibit DPPH radical formation by $50 \%$ ) values of the samples.

\subsection{Antibacterial Activity}

\subsubsection{Bacterial strains}

Antimicrobial activity of the crude plant extract as well as biosynthesized AgNPs was evaluated by calculating zone of inhibition against both gram-positive $S$. aureus (ATCC 25923) and gram-negative E. coli (ATCC 25922)bacterial strains.

\subsubsection{Inoculation}

A pure colony of each bacterial strain was inoculated in the $5 \mathrm{~mL}$ nutrient broth till the growth was equivalent with MacFarland standard $(0.5 \%)$ as recommended by WHO. The inoculated culture bottles were kept in the incubator at $37{ }^{\circ} \mathrm{C}$ for $3-4 \mathrm{hrs}$. The turbidity of the sub-cultured bacterial suspension was adjusted at freshly prepared 0.5 McFarland standards. These bacterial inoculums were used for swabbing on the MHA plates to test the antibacterial potential of the AgNPs.

\subsubsection{Agar well diffusion method}

The well diffusion method using Muller Hinton agar (MHA) medium was applied to calculate the antibacterial activity of both the crude extract and synthesized AgNPs samples. The required number of wells was built in MHA using cork borer (6 $\mathrm{mm}$ diameter). Inoculums containing $10^{6} \mathrm{CFU} / \mathrm{mL}$ of bacteria were spread with a sterilized cotton swab on the MHA medium plates. $20 \mu \mathrm{L}$ each extract of the concentration $100,50,25$ and $12.5 \mathrm{mg} / \mathrm{mL}$ was added in the wells and same volume of extraction solvent i.e methanol and DMSO was used as negative control. $1 \mathrm{mg} / \mathrm{mL}$ of streptomycin was taken as positive control. Similarly, $20 \mu \mathrm{L}$ of AgNPs prepared from different aqueous plant extract was filled in the well where aqueous plant extract was taken as negative control and $1 \mathrm{mg} / \mathrm{mL}$ of 
streptomycin was taken as positive control. Plates were left till the extract diffused into the medium, covered the plate and incubated at $37^{\circ} \mathrm{C}$ for $24 \mathrm{~h}$. After overnight incubation, the diameter of the zone of inhibition (ZOI) in $\mathrm{mm}$ were measured (Regmi et al. 2019). The samples showing ZOI greater or equal to $8 \mathrm{~mm}$ in diameter were considered that the samples were effective as antibacterial agent.

\section{RESULTS AND DISCUSSION}

\subsection{Plant Materials}

Plants having high medicinal values used locally as a medicine for the remedy of the spectrum of illness were selected for the study. Bioactive compounds possessing pharmacological activity were extracted by using methanol as solvent through soxhlet extractor. The yield of B. asiatica was obtained in $15.5 \%$ while C. fistula yield was $12.8 \%$. Climatic conditions, parts of the plant used, extraction time, temperature and extraction procedure play apivotal role in the isolation of bioactive compounds

\subsection{UV-vis Spectra Analysis of AgNPs}

An UV-visible spectrum is a well-known technique to prove the formation of metal nanoparticles provided surface plasmon resonance exists for the metal nanoparticles. Having free electors on metal nanoparticle produces the band of surface plasmon resonance (SPR) because of the high energy electronic vibration in the resonance. Changing color from yellow to brown informed the synthesis of AgNPs. The spectrum displays the vital role of $\mathrm{AgNO}_{3}$ along with the presence of plant constituents in the formation of AgNPs. The AgNPs of plants B. asiatica and $C$. fistula extracts showed the UV absorption peak at the range of 400-450 nm. B. asiaticaand C. fistula exhibited the peak at $450 \mathrm{~nm}$ and $445 \mathrm{~nm}$ respectively in the UV-Vis spectroscopy (Fig.1). The spectra demonstrated a gradual decrease of the absorbance from the wavelength from 450 to $380 \mathrm{~nm}$. The occurrence of the silver SPR band in the range of 400-450 nm indicates the formation of AgNPs (El-Shanshoury et al. 2011;Umoren et al. 2014; Vijay Kumar et al. 2014).

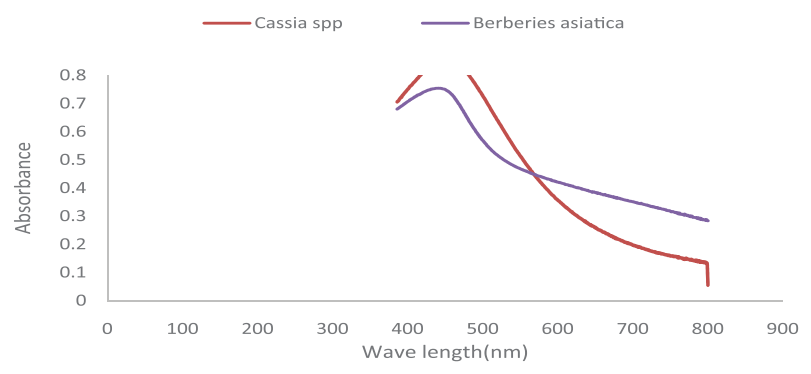

Fig. 1. UV-Vis spectra showing surface Plasmon resonace of biosynthesized AgNPs of the plants.

\subsection{FTIR Spectroscopic Analysis}

The AgNPs in powder form was used for the analysis of FTIR to verify the presence of functional groups which are essential for the reduction of AgNPs from the plant samples. FTIR analysis of the AgNPs was performed in the range of $4000-500 \mathrm{~cm}^{-1}$. Upon performing FTIR, it was observed that the AgNPs showed the peak at the range of $3400-3700 \mathrm{~cm}^{-1}$ due to the presence hydroxyl group (-OH) and at the range of $1690-1630 \mathrm{~cm}^{-1}$ shows the presence of carbonyl group $(-\mathrm{C}=\mathrm{O})$, which were mainly responsible for bio-reduction of silver nitrate and synthesis of its nanoparticles. The absorption spectrum of B. asiaticasynthesized AgNPs (Fig. 2) recordedthe major peak at $3442 \mathrm{~cm}^{-1}$ due to O-H stretching vibration in alcohol and phenol and the peak at $1633 \mathrm{~cm}^{-1}$ suggests the presence of carbony groups. The AgNPs of C. fistula showed the dominant peaks at $3441 \mathrm{~cm}^{-1}$ due to $\mathrm{O}-\mathrm{H}$ stretching vibration in alcohol and phenol and at $1635 \mathrm{~cm}^{-1}$ due to the presence of carboxy groups. The Fig. 2 and Fig. 3 indicate the presence of many fundamental groups involved in conversion of silver ions to silver nanoparticles.

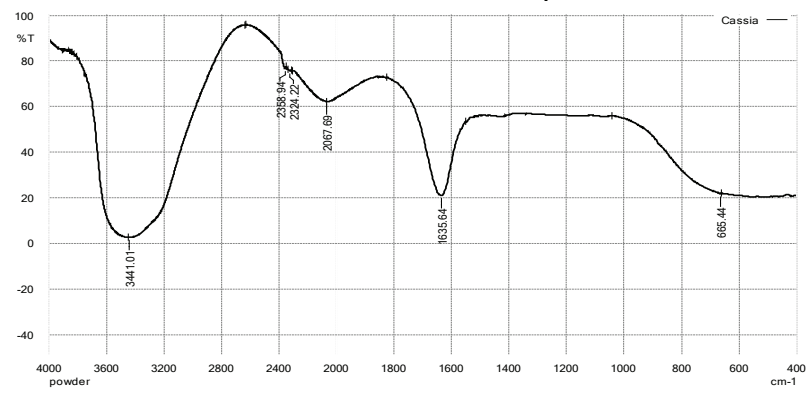

Fig. 2. FTIR spectrum of the AgNPs synthesized from $B$. asiatica (chutro)

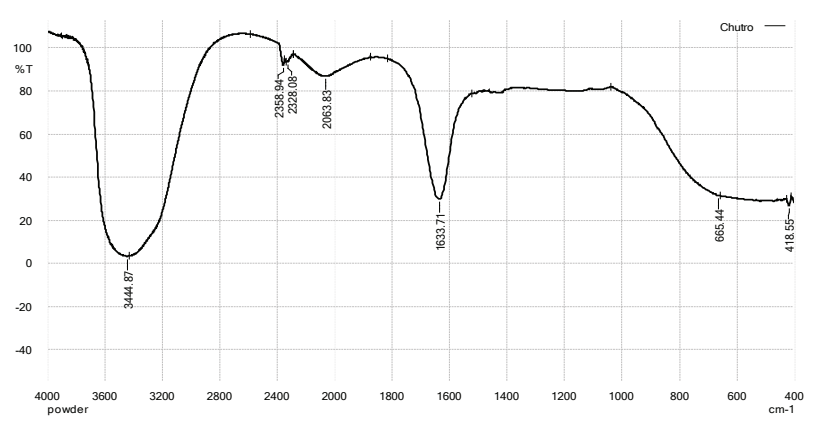

Fig.3. FTIR spectrum of the AgNPs synthesized from C.fistula

\subsection{XRD Analysis}

The crystallize size and structure of AgNPs was studied by using XRD. AgNPs of B. asiatica (Fig. 4) observed diffraction peak in XRD at $2 \theta=32.1,38.3,44.5,64.5$ and 77.5 and C. fistula (Fig. 5) demonstrated the dominant 
peaks at 29.1, 38.1, 43.7, 64.3 and 77.3. These found XRD peaks confirmed that biosynthesized AgNPs nanocrystal and crystalline in sturcture where the peaks corresponded with standard. The peaks seen in XRD can be attributed to the planes (122), (111), (200), (220) and (311) facet of silver crystal respectively (Roy et al. 2015). From the XRD patterns, peak intensity, position and full width at half maximum (FWHM) were analyzed using DebyeScherrer's formula to determine the crystallite size. Some peaks in the spectra couldn't assign due to the presence of some bioorganic compounds/protein(s) in the extracts and crystallizes on the surface of the silver metal (Philip 2011). The AgNPs particle in crystalline structure was calculated applying Debye-Scherrer's equation (Ajitha et al. 2014):

$\mathrm{D}=0.9 \lambda / \beta \operatorname{Cos} \theta$ where, $\lambda=$ wavelength of the $\mathrm{x}$-ray

$\beta=$ broadening of the diffraction line measured as half of its maximum intensity in radians and

$\theta=$ Bragg's diffraction angle

The crystalline size of the AgNPs was estimated from the line width of the (111) XRD peak of the both samples. The AgNPs particle size of B. asiatica and C. fistula was found 13 and $15 \mathrm{~nm}$ respectively.

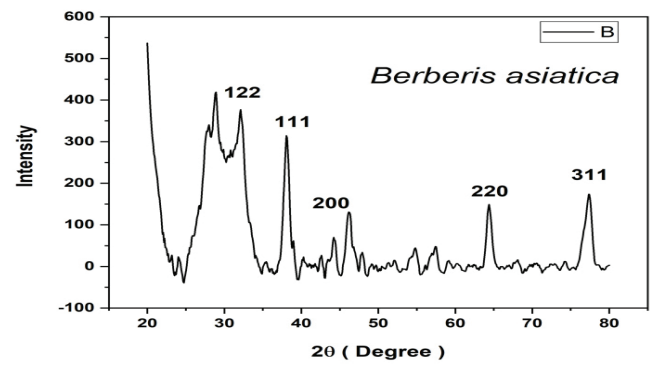

Fig. 4. XRD pattern of the AgNPs synthesized from $B$. asiatica.

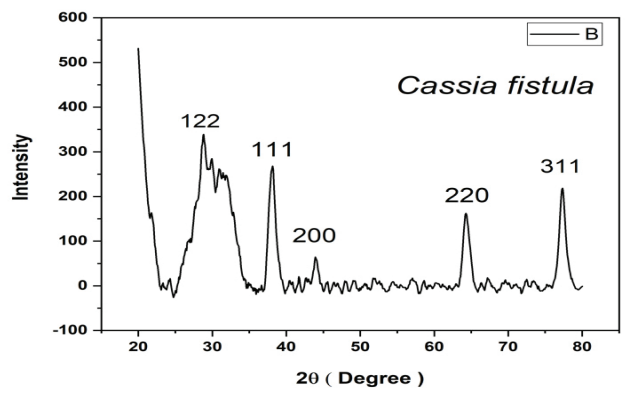

Fig. 5. XRD pattern of the AgNPs synthesized from $C$. fistula.

\subsection{Antioxidant Activity of AgNPs}

The biogenic synthesis of AgNPs using $B$. asiatica exhibited significant antioxidant potential having $\mathrm{IC}_{50} 65.1 \pm 1.30 \mu \mathrm{g} /$ $\mathrm{mL}$ but crude methanolic extract showed130.4 $\pm 0.12 \mu \mathrm{g} /$
mL. Similarly, the AgNPs of C. fistula had antioxidant activity of $\mathrm{IC}_{50} 100.2 \pm 0.82 \mu \mathrm{g} / \mathrm{mL}$ but respective crude extract possessed significantly higher $\mathrm{IC}_{50}$ (Table 2 ). Many papers have been published reporting potential antioxidant activity of green synthesized AgNPs from $P$. pinnate (Priya et al. 2016) and E. suberosa (Yugal K. Mohanta et al. 2017). The results concluded that the implication of AgNPS as a natural antioxidant is more effective in the comparison of its crude extract for health protection from different oxidative stress linked with degenerative diseases. AgNPs can be used in animal models and also in clinical trial after evaluating their antioxidant activity.

Table 2. Antioxidant activity $\left(\mathrm{IC}_{50}\right)$ of AgNPs and crude extract of the medicinal plants.

\begin{tabular}{cccc}
\hline S.N. & Plant species & $\begin{array}{c}\mathbf{I C}_{\mathbf{5 0}} \mathbf{M e O H} \text { extract } \\
(\boldsymbol{\mu g} / \mathbf{m L})\end{array}$ & $\begin{array}{c}\mathbf{I C}_{\mathbf{5 0}} \mathbf{A g N P s} \\
(\boldsymbol{\mu} \mathbf{g} / \mathbf{m L})\end{array}$ \\
\hline 1. & B. asiatica & $130.4 \pm 0.12$ & $65.1 \pm 1.30$ \\
2. & C. fistula & $170.5 \pm 1.12$ & $100.2 \pm 0.82$ \\
\hline
\end{tabular}

\subsection{Antibacterial Activity of AgNPs}

Plant based AgNPs are used extensively in the field of medicine, health and environment. We examined the antibacterial potential of AgNPs by calculating the zone of inhibition against both gram-positive and gram-negative bacteria. The details are shown in Fig. 6 and Table 3.The maximum ZOI exhibited by the AgNPs of $B$. asiatica against $S$. aureus was $15 \mathrm{~mm}$ depicted in Fig. 6(A) and the minimum inhibition less than $8 \mathrm{~mm}$ was performed by the AgNPs of $R$. australe against $K$. pneumonia shown in Fig. 6(C). The findings indicated that the biosynthesized AgNPs is more effective in the comparison to the crude extract because AgNPs contains large surface area that facilitates ameliorating contact with bacterial cell wall (Ibrahim 2015). Many similar results were published previously explaining inhibition of AgNPs against $E$. coli using Menthapiperita (Mubarak Ali et al. 2011) and Acalyphaindica (Krishnaraj et al. 2010).

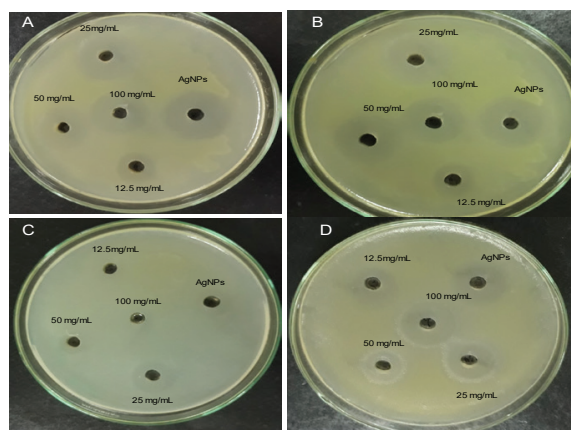

Fig. 6. Antimicrobial activity of different concentration of crude extract and AgNPs of B. asiatica 
(A) S. aureus, (B) E. coli and C. fistula (C) S. aureus and (D) E. coli.

The mechanism behind the antimicrobial activity of AgNPs is established as the electrostatic attraction between the positive charges on the $\mathrm{Ag}^{+}$ion and the negative charges developed on the bacterial cell wall due to phospholipidsbilayers (Raut et al. 2010). The working process of AgNPs against microorganisms is really intriguing. In the first step, AgNPs attaches on the cell wall and membrane then penetrate inside the cells and start damaging inner structures of the microorganisms. In the third step, nanoparticles stimulate cellular toxicity and oxidative stress by producing reactive oxygen species. Finally, error is occurred in the signal transduction pathway and organism remains no longer alive (Tian et al. 2017).

Table 3. Zone of Inhibition ( $\mathrm{mm}$ ) of plant extracts and nanoparticles in gram positive ( $S$. aureus) and gram negative (E. coli) bacteria.

\begin{tabular}{c|c|c|c}
\hline Plants & Concentration & \multicolumn{2}{|c}{ Zone of inhibition $\mathbf{( m m )}$} \\
\cline { 3 - 4 } & $\mathbf{( 1 2 . 5} \mathbf{~ m g} / \mathbf{m L})$ & S. aureus & E. coli \\
\hline \multirow{2}{*}{ B. asiatica } & Crude & 9 & 7 \\
\cline { 2 - 4 } & AgNPs & 15 & 14 \\
\hline \multirow{2}{*}{ C. fistula } & Crude & 6 & 8 \\
\cline { 2 - 4 } & AgNPs & 7 & 12 \\
\hline
\end{tabular}

\section{CONCLUSION}

The green synthesis of AgNPs were performed by using methanolic extract of medicinal plants B. Asiatic and $C$. fistula. The synthesized AgNPs were characterized by UVVis spectrophotometer demonstrating wavelength at 400$450 \mathrm{~nm}$. FTIR data supported the presence of functional groups by appearing the peak at the range $3400-3700 \mathrm{~cm}^{-1}$ of the hydroxyl group (O-H) and the peak at 1690-1630 $\mathrm{cm}^{-1}$ of the carbonyl group $(\mathrm{C}=\mathrm{O})$. XRD analysis predicted that biosynthesized AgNPs nanocrystal and crystalline in sturcture were the sample peaks corresponded with standard. AgNPs prepared from B. asiatica showed more potent antioxiant activity $\left(\mathrm{IC}_{50} 65.1 \pm 1.3 \mu \mathrm{g} / \mathrm{mL}\right)$ than the others. AgNPs of $B$. asiatica demonstrated more effective antibacterial property against $S$. aureus $(15 \mathrm{~mm})$ among all others. Therefore, biosynthesized AgNPs is more valuable than their corresponding crude extracts. These green synthesized AgNPs would be applied in different medical sectors in near future.

\section{ACKNOWLEDGEMENT}

The Authors would like to express their gratitude to Dr. Rosa Ranjit (Senior scientist, NAST) for accessing Elisa microplate reader, Dr. Deependra Das Mulmi (Senior scientist, NAST) for UV-Vis spectrophotometer, Dr. Ram Chandra Poudel (Senior scientist, NAST) for plant identification, Mr. Hari Ram Shrestha (NAST) for the XRD data, Ms. Bima Maharjan (ARF, NAST) for the FTIR data and Mr. Dilip Karki (Physical Science Laboratory, NAST) for the XRD data analysis and Nepal Academy of Science and Technology for providing financial supports, time and space.

\section{REFERENCES}

1. Ahmed, S., M. Ahmad Saifullah, B.L. Swami \& S. Ikram. 2016. Green synthesis of silver nanoparticles using Azadirachta indica aqueous leaf extract . Journal of Radiation Research and Applied Sciences, 9(1), 1-7.

2. Ajitha, B., K.R.Y. Ashok \& R.P. Sreedhara. 2014. Biogenic nano-scale silver particles by Tephrosia purpurea leaf extract and their inborn antimicrobial activity. Spectrochim Acta Part A, 121, 164-172.

3. Belwal, T., I.D. Bhatt, R.S. Rawal \& V. Pande. 2017. Microwave-assisted extraction (MAE) conditions using polynomial design for improving antioxidant phytochemicals in Berberis asiatica Roxb. ex DC. leaves. Industrial Crops and Products, 95, 393-403.

4. Beyene, H.D., A.A. Werkneh, H.K. Bezabh, \& T.G. Ambaye. 2017. Synthesis paradigm and applications of silver nanoparticles (AgNPs), a review. Sustainable Materials and Technologies, 13, 18-23.

5. Bhandari, D.K., G. Nath, A.B. Ray \& P.V. Tewari. 2000. Antimicrobial activity of crude extracts from Berberis asiatica stem bark. Pharmaceutical Biology, 38(4), 254-257.

6. Bhardwaj, D. \& N. Kaushik. 2012. Phytochemical and pharmacological studies in genus Berberis. Phytochemistry Reviews, 11(4), 523-542.

7. Dipankar, C. \& C. Murugan. 2012. The green synthesis, characterization and evaluation of the biological activities of silver nanoparticles synthesized from Iresine herbstii leaf aqueous extracts. Colloids and Surfaces B: Biointerfaces, 98, 112-119.

8. El-Shanshoury, A.E.-R.R., S.E. ElSilk, \& M.E. Ebeid. 2011. Extracellular biosynthesis of silver nanoparticles using Escherichia coli ATCC 8739, Bacillus subtilis ATCC 6633, and Streptococcus thermophilus ESh1 and their antimicrobial activities.ISRNNanotechnology, 2011, 1-7. 
9. Elamawi, R.M., R.E. Al-Harbi \& A.A. Hendi. 2018. Biosynthesis and characterization of silver nanoparticles using Trichoderma longibrachiatum and their effect on phytopathogenic fungi. Egyptian Journal of Biological Pest Control, 28(1), 1-11.

10. Ibrahim, H.M.M. 2015. Green synthesis and characterization of silver nanoparticles using banana peel extract and their antimicrobial activity against representative microorganisms. Journal of Radiation Research and Applied Sciences, 8(3), 265-275.

11 Iravani, S., H. Korbekandi, S.V. Mirmohammadi \& B. Zolfaghari. 2014. Synthesis of silver nanoparticles: Chemical, physical and biological methods. Research in Pharmaceutical Sciences, 9(6), 385-406).

12. Khandel, P., R.K. Yadaw, D.K. Soni, L. Kanwar \& S.K. Shahi. 2018. Biogenesis of metal nanoparticles and their pharmacological applications: present status and application prospects. Journal of Nanostructure in Chemistry, 8(3), 217-254.

13. Kharissova, O.V., B.I. Kharisov, C.M.O. González, Y.P. Méndez \& I. López. 2019. Greener synthesis of chemical compounds and materials. Royal Society Open Science, 6(11), 1-41.

14. Krishnaraj, C., E.G. Jagan, S. Rajasekar, P. Selvakumar, P.T. Kalaichelvan \& N. Mohan. 2010. Synthesis of silver nanoparticles using Acalypha indica leaf extracts and its antibacterial activity against water borne pathogens. Colloids and Surfaces B: Biointerfaces, 76(1), 50-56.

15. Loo, Y.Y., Y. Rukayadi, M.A.R. Nor-Khaizura, C.H. Kuan, B.W. Chieng, M. Nishibuchi \& S. Radu. 2018. In Vitro antimicrobial activity of green synthesized silver nanoparticles against selected Gram-negative foodborne pathogens. Frontiers in Microbiology, 9(7), $1-7$.

16. Marambio-Jones, C. \& E.M.V. Hoek. 2010. A review of the antibacterial effects of silver nanomaterials and potential implications for human health and the environment. Journal of Nanoparticle Research 12(5), 1531-1551.

17. Mittal, A.K., Y. Chisti \& U.C. Banerjee. 2013. Synthesis of metallic nanoparticles using plant extracts. Biotechnology Advances, 31(2) 346-356.

18. Mohanta, Yugal K., S.K. Panda, R. Jayabalan, N. Sharma, N., A.K. Bastia \& T.K. Mohanta. 2017. Antimicrobial, antioxidant and cytotoxic activity of silver nanoparticles synthesized by leaf extract of
Erythrina suberosa (Roxb.). Frontiers in Molecular Biosciences, 4(3), 1-9.

19. Mohanta, Yugal Kishore \& S.K. Behera. 2014. Biosynthesis, characterization and antimicrobial activity of silver nanoparticles by Streptomyces $s p$. SS2. Bioprocess and Biosystems Engineering, 37(11), 2263-2269.

20. Mohanta, Yugal Kishore, S.K. Panda, K. Biswas, A. Tamang, Bandyopadhyay, J., De, D., D. Mohanta \& A.K. Bastia. 2016. Biogenic synthesis of silver nanoparticles from Cassia fistula (Linn.): In vitro assessment of their antioxidant, antimicrobial and cytotoxic activities. IET Nanobiotechnology, 10(6), 438-444.

21. MubarakAli, D., N. Thajuddin, K. Jeganathan \& M. Gunasekaran. 2011. Plant extract mediated synthesis of silver and gold nanoparticles and its antibacterial activity against clinically isolated pathogens. Colloids and Surfaces B: Biointerfaces, 85(2), 360-365.

22. Nagaich, U., N. Gulati \& S. Chauhan. 2016. Antioxidant and antibacterial potential of silver nanoparticles: Biogenic synthesis utilizing Apple extract. Journal of Pharmaceutics, 2016, 1-8.

23. Nayak, D., S. Ashe, P.R. Rauta, M. Kumari \& B. Nayak. 2016. Bark extract mediated green synthesis of silver nanoparticles: Evaluation of antimicrobial activity and antiproliferative response against osteosarcoma. Materials Science and Engineering C, 58, 44-52.

24. Philip, D. 2011. Mangifera Indica leaf-assisted biosynthesis of well-dispersed silver nanoparticles. Spectrochimica Acta - Part A: Molecular and Biomolecular Spectroscopy, 78(1), 327-331.

25. Priya, R.S., D. Geetha \& P.S. Ramesh. 2016. Antioxidant activity of chemically synthesized AgNPs and biosynthesized Pongamia pinnata leaf extract mediated AgNPs - A comparative study. Ecotoxicology and Environmental Safety, 134, 308-318.

26. Raut, R.W., N.S. Kolekar, J.R. Lakkakula, V.D. Mendhulkar \& S.B. Kashid. 2010. Extracellular synthesis of silver nanoparticles using dried leaves of Pongamia pinnata (L) pierre. Nano-Micro Letters, 2(2), 106-113.

27. Regmi, R., M. Shrestha, M.R. Banjara \& D. Khadka. 2019. Antidiabetic and antimicrobial properties of some high altitude medicinal plants of Nepal. Proceedings of the Pakistan Academy of Sciences: Part B, 56(3B), 69-74. 
28. Roy, K., C.K. Sarkar \& C.K. Ghosh. 2015. Plantmediated synthesis of silver nanoparticles using parsley (Petroselinum crispum) leaf extract: spectral analysis of the particles and antibacterial study. Applied Nanoscience (Switzerland), 5(8), 945-951.

29. Satyavani, K., T. Ramanathan \& S. Gurudeeban. 2011. Plant mediated synthesis of biomedical silver nanoparticles by using leaf extract of Citrullus colocynthis. Research Journal of Nanoscience and Nanotechnology, 1(2), 95-101.

30. Tian, J., K. K. Wong, C.M. Ho, C.N. Lok, W.Y. Yu \& C.M. Che. 2007. Topical delivery of silver nanoparticles promotes wound healing. Chemistry Medicinal Chemistry, 2, 129-136.
31. Umoren, S. A., I.B. Obot \& Z.M. Gasem. 2014. Green synthesis and characterization of silver nanoparticles using red apple (malus domestica) fruit extract at room temperature. Journal of Materials and Environmental Science, 5(3), 907-914.

32. Vijay Kumar, P.P.N., S.V.N. Pammi, P. Kollu, K.V.V. Satyanarayana \& U. Shameem. 2014. Green synthesis and characterization of silver nanoparticles using Boerhaavia diffusa plant extract and their anti bacterial activity. Industrial Crops and Products, 52, 562-566. 